\title{
The prelimbic cortex directs attention toward predictive cues during fear learning
}

\author{
Melissa J. Sharpe and Simon Killcross \\ School of Psychology, University of New South Wales Australia, Kensington, New South Wales 2052, Australia
}

\begin{abstract}
The prelimbic cortex is argued to promote conditioned fear expression, at odds with appetitive research implicating this region in attentional processing. Consistent with an attentional account, we report that the effect of prelimbic lesions on fear expression depends on the degree of competition between contextual and discrete cues. Further, when competition from contextual cues is low, we found that PL inactivation resulted in animals expressing fear toward irrelevant discrete cues; an effect selective to inactivation during the learning phase and not during retrieval. These data demonstrate that the prelimbic cortex modulates attention toward cues to preferentially direct fear responding on the basis of their predictive value.
\end{abstract}

Learning is selective to cues which have most reliably signaled an outcome in the past. Restricting learning to predictive cues relies on the ability to preferentially attend toward cues that are better predictors of an event (Mackintosh 1975; Le Pelley 2004). In appetitive procedures, manipulation of prelimbic (PL) activity impairs extra-dimensional set shifting, response set shifting, and utilizing contextual cues to change the degree of attention allocated toward discriminative stimuli (Birrell and Brown 2000; Floresco et al. 2008 Marquis et al. 2007). Recently, this has been characterized as a specific deficit in modulating the degree of attention directed toward cues on the basis of their associative history (Sharpe and Killcross 2014). Thus, the PL cortex is argued to be the neural locus of changes in attention toward cues on the basis of how well they predict an outcome, restricting learning to cues which are best predictors of an outcome (Sharpe and Killcross 2014).

However, findings that the degree of PL activity correlates with the magnitude of a conditioned response to fearful stimuli have been interpreted as a role for this region in the expression of conditioned responding (Corcoran and Quirk 2007; BurgosRobles et al. 2009; Sierra-Mercado et al. 2011). This is despite research demonstrating that manipulation of PL functioning during aversive procedures has produced equivocal results. For example, pretraining lesions of the dorsal medial prefrontal cortex, predominantly localized to PL cortex, have been shown to enhance fear conditioning to both contextual and discrete stimuli (Morgan and LeDoux 1995), enhance learning about a context at the expense of a CS (Lacroix et al. 2000), or have no effect (Holson 1986).

These differences may be accounted for by appealing to the role of the PL cortex in directing attention toward predictive cues. More specifically, differences in parameters used in these studies may produce a differential degree of competition between contextual and discrete cues. It is well established that both a context and a discrete CS will compete to become associated with an unconditioned stimulus (US), such as a shock. The degree to which these stimuli will become associated with the US is dependent how well they predict it (Rescorla 1984; Mais and Vossen 1993). For example, pairing a brief discrete stimulus with presentation of shock will often substantially overshadow learning about the background context as it more accurately predicts shock delivery (Rescorla 1984; Mais and Vossen 1993). The ability to demonstrate greater learning about the more accurate predictor

\section{Corresponding author: m.sharpe@unsw.edu.au}

Article is online at http://www.learnmem.org/cgi/doi/10.1101//m.038273.115. is attributable in part to the ability of animals to change the degree of attention directed toward all present cues on the basis of how well they predict an outcome (Mackintosh 1975). Thus, the discrete stimulus will receive greater attention relative to the context because it is the better predictor of US delivery, and so will be learned about preferentially.

An involvement of the PL cortex in using attentional modulation to resolve competition between a discrete stimulus and a context will mean that changing the degree of competition between discrete and contextual cues should alter the effects of PL lesions. There are many factors that can influence the degree of competition between discrete and contextual cues. For example, Mais and Vossen (1993) found that preexposing a context prior to conditioning in the absence of any conditioned or unconditioned stimuli resulted in substantially less conditioning to the context, making the CS a better predictor of the shock. There are also a number of reports demonstrating that lengthening the intertrial interval (ITI) between successive CS-US pairings increases learning about the CS relative to the context (McAllister et al. 1974; Holland 2000). In line with a role for the PL cortex in resolving any such competition, studies in which animals have received considerable pretraining exposure to the context (and therefore reduced CS-context competition) have found no effect of PL lesions on fear conditioning (Holson 1986). In contrast, studies which have used less preexposure to the context and shorter ITIs (which promotes competition between contextual and discrete cues) have found that animals with PL lesions (relative to sham-lesioned animals) exhibit enhanced learning about a context (Morgan and LeDoux 1995; Lacroix et al. 2000), in one instance at the expense of learning about a CS (Lacroix et al. 2000). Findings that the effects of PL lesions may vary with the degree of competition between discrete and contextual cues is consistent with a view that the PL cortex may play a role in modulating attention toward cues according to how well they predict an outcome in an aversive setting, paralleling the appetitive literature.

In order to test this hypothesis directly, we examined the impact of pretraining lesions of the PL cortex on the ability of animals to resolve competition between discrete and contextual cues. We systematically varied the length of ITIs and the degree

(C) 2015 Sharpe and Killcross This article is distributed exclusively by Cold Spring Harbor Laboratory Press for the first 12 months after the full-issue publication date (see http://learnmem.cshlp.org/site/misc/terms.xhtml). After 12 months, it is available under a Creative Commons License (Attribution-NonCommercial 4.0 International), as described at http://creativecommons.org/ licenses/by-nc/4.0/. 
of pretraining exposure to the context to change the level of competition between these cues. In Experiment 1a, we subjected rats with PL lesions ( $n=8$; Fig. $1 \mathrm{~A})$ and sham lesions ( $n=8$; Fig. $1 \mathrm{~A})$ to a fear conditioning procedure with parameters designed to promote high competition between discrete and contextual cues. Excitotoxic lesions were produced with bilateral infusions of $0.35 \mu \mathrm{L}$ of a $0.067 \mathrm{M} \mathrm{N}$-methyl-D-aspartate (NMDA) as described previously (Sharpe and Killcross 2014). The PL region was defined by the boundaries described by Paxinos and Watson (1998), where rats exhibiting incomplete damage to the PL cortex, or with extensive damage to surrounding areas, were excluded from all analyses. On day 1, rats were placed in a novel context for 3 min prior to the delivery of the first of three $70-\mathrm{dB}$ tone-shock pairings where a 1-sec $0.8 \mathrm{~mA}$ shock US was delivered at the termination of 30-sec CS presentations. CS-shock presentations were separated by an ITI, which varied about a 64 -sec mean. The following day, rats were then tested for levels of freezing toward the context in a 10-min session. Freezing was defined as the absence of all movement except respiration. Each rat was scored as "freezing" or "not freezing" by a blind, trained observer, every 2 sec during presentation of the CS and a 30-sec baseline period that immediately preceded CS presentation (i.e., pre-CS period). The number of observations where animals spent freezing relative to the total number of observations was then transformed into a percentage score for analyses. In the 10-min test in the conditioning context, we found that rats with PL lesions exhibited higher levels of freezing toward the context relative to sham-lesioned controls (see Fig. 2A; $\left.F_{(1,15)}=6.15, P<0.05\right)$. On day 3 , we placed rats back into the conditioning context and presented the CS five times in the absence of shock, according to parameters used during conditioning. Though animals with PL lesions exhibited higher levels of fear toward the CS, this difference was not statistically significant (see Fig. $\left.2 \mathrm{~A} ; F_{(1,15)}=1.55, P>0.05\right)$. This CS test was conducted in
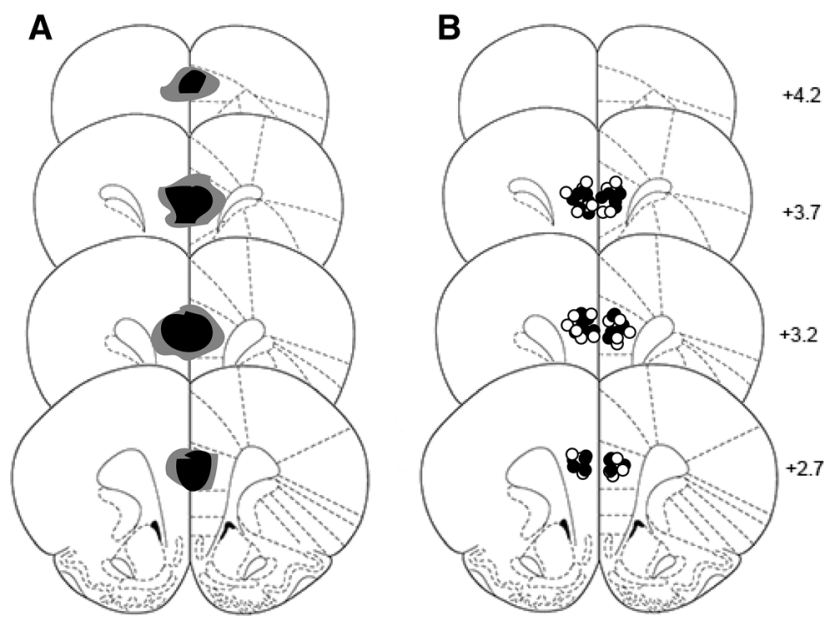

Figure 1. Schematic representations of excitotoxic lesions and cannulas placement for Experiments 1 and 2. Coronal sections are taken from the following points on the anteroposterior plane beginning at top: +4.20 , +3.70 , +3.20, and $+2.70 \mathrm{~mm}$ anterior to bregma (Paxinos and Watson 1998). (A) Shaded areas represent the maximum (dark gray) and minimum (black) extent of the lesions for the animals that were included in behavioral analyses for Experiment 1. Damage typically extended from +4.20 to $+2.70 \mathrm{~mm}$ anterior to bregma. Subjects with significant damage to the adjacent anterior cingulate or infralimbic cortices were excluded from all analyses. (B) Placement of cannulas tips for Experiment 2. All cannulas placements feel between +3.7 and +2.7 $\mathrm{mm}$ anterior to bregma. Animals with placements considered outside the boundaries of the PL cortex (Paxinos and Watson 1998), were excluded from all analyses. the conditioning context. Given animals with PL lesions exhibit greater levels of fear toward the context following conditioning, responding to the CS may reflect the associative strength of both the contextual and discrete cues. Thus, in Experiment $1 \mathrm{~b}$ we trained a separate group of rats with either PL lesions $(n=7$; Fig. 1A) or sham lesions ( $n=6$; Fig. $1 \mathrm{~A})$ using the same parameters as before. However, we tested levels of fear toward the CS in a distinct but familiar context. Here, we found that animals with PL lesions exhibited lower levels of fear toward the CS (see Fig. 2B; $\left.F_{(1,11)}=5.72, P<0.05\right)$. The lower levels of conditioned fear to the CS in PL-lesioned animals in Experiment 1b, together with the results of Experiment 1a (which used identical parameters), indicates that the PL region is not responsible for mediating expression of fear to a CS per se, but rather for competition between cues during conditioning. When competition between contextual and discrete cues is high, animals with PL lesions exhibit higher levels of fear toward contextual cues at the expense of learning about the predictive CS.

In the Experiment 1c, we examined the impact of PL lesions on the ability of animals to exhibit fear toward a CS when competition between discrete and contextual cues is low. Here, we gave experimentally naïve animals with PL lesions ( $n=13$; Fig. $1 \mathrm{~A})$ or sham lesions ( $n=15$; Fig. $1 \mathrm{~A})$ two 1-h sessions of preexposure to both the conditioning and test contexts prior to conditioning. During conditioning, rats were placed in the conditioning context for $\sim 8 \mathrm{~min}$ prior to the first of three tone-shock pairings where the shock US was delivered at the termination of 30-sec CS presentations. CS-shock pairings were separated by an ITI, which varied about an 8-min mean. Rats remained in the conditioning chambers for an additional 8 min after presentation of the final tone-shock pairing. Thus, during these conditioning sessions we varied the parameters to reduce competition from contextual cues (by preexposing the animals to the context and used lengthy ITIs, where pilot studies had shown that these parameters produce the same amount of conditioning toward the CS as those used in the previous experiment). Subsequently, we tested rats for levels of freezing toward the CS in the test context and found that animals with PL lesions exhibited high levels of fear toward the CS, comparable with sham-lesioned animals (see Fig. 2C; $F<1$ ). Low levels of pre-CS freezing during the test session revealed that animals did not acquire fear to the context (mean \% [ \pm SEM]: sham 2.27 [1.03]; PL 1.64 (0.60), $F<1$ ). These data demonstrate that animals with lesions of the PL cortex are able to exhibit fear toward a CS when competition from contextual cues is low and, thus, modulation of attention toward the predictive CS is not necessary.

In order to confirm a role of the PL cortex in modulating attention toward cues during fear learning (rather than any specific deficit in learning about contextual cues), we looked at the impact of PL inactivation during conditioning in an overshadowing procedure. Overshadowing involves presenting two cues as an audiovisual compound followed by shock. When the visual element of the compound is presented alone in an extinction test session, responding to that stimulus is lower relative to responding toward a visual stimulus that paired with the outcome individually during conditioning. Overshadowing occurs because the less salient visual element of the compound is rendered redundant by the presence of the more salient auditory stimulus and attention declines toward it (Mackintosh 1975, 1976; Le Pelley 2004; Pearce and Mackintosh 2010). In the overshadowing procedure, we used conditioned suppression as an index of fear (according to the formula $\mathrm{A} /(\mathrm{A}+\mathrm{B})$ ) as pilot studies had demonstrated that assessing change in lever-press responding was a more sensitive measure when assessing levels of fear toward multiple CSs (in comparison to the use of one CS in the previous studies). Following extensive preexposure to the context during lever-press 
training which we have described in detail elsewhere (Sharpe and Killcross 2015), rats were given one stimulus preexposure session where they were given two presentations of each stimulus individually. This was to reduce any unconditioned suppression or generalization across the stimuli prior to conditioning (but not enough to produce latent inhibition; Rhodes and Killcross 2007). Following preexposure, we subjected rats to two conditioning sessions. During conditioning, two stimuli were presented simultaneously to form an audio-visual compound and two other cues, one auditory and one visual, were presented individually. The order of presentation of stimuli during conditioning and test was fully counterbalanced. The four stimuli consisted of click, noise, flashing panel lights, and a house light, counterbalanced across rats within modality (for more details, see Sharpe and Killcross 2014). Each conditioning session consisted of two 30 -sec presentations of each stimulus followed by a $0.5 \mathrm{~mA}$ footshock. Stimulus presentations were separated by a variable ITI averaging about a 7-min mean to further reduce competition from contextual cues. Following conditioning, rats were tested for levels of fear acquired toward the CSs in two extinction test sessions where each session comprised two presentations of each stimulus all presented individually in a fully counterbalanced fashion. In Experiment 2a, we found all rats initially exhibited suppression of lever pressing during the first presentation of the to-be conditioned stimuli during the preexposure session (mean suppression ratio $[ \pm$ SEM] : saline 0.22 [0.05]; muscimol 0.25 [0.03]), which reduced on the second presentation (mean suppression ratio [ \pm SEM]: saline 0.41 [0.06]; muscimol 0.47 [0.07]). Further, during conditioning we found no effect of the bilateral $0.5 \mu \mathrm{g} / \mu \mathrm{L}$ dose of muscimol ( $n=12$; Fig. 1B), previously found to produce effects selective to the PL cortex (Marquis et al. 2007), or saline $(n=11$; Fig. 1B) on responding. All rats gradually suppressed lever-press responding during CS presentations, so that by the second conditioning session animals had stopped responding during the CS presentations (mean suppression ratio $[ \pm$ SEM]: saline 0.09 [0.06]; muscimol 0.05 [0.04]). During the extinction test sessions, we found that animals infused with either muscimol or saline during the conditioning session exhibited high levels of fear toward both auditory cues, regardless of whether they were trained in compound or as an element (mean suppression ratio [ \pm SEM]: saline, compound 0.02 [0.02] element 0.12 [0.10]; muscimol, compound 0.03 [0.03] element 0.00 [0]). However, we found that rats receiving saline infusions exhibited overshadowing with respect to the visual stimuli, demonstrating less suppression toward the visual stimulus trained in compound relative to higher levels of suppression toward the visual stimulus paired with the US individually. In contrast, animals without PL function failed to exhibit this effect. This was confirmed by statistical analyses where a mixed-design repeated-measures ANOVAs on responding to the visual stimuli revealed a significant interaction between the overshadowing effect and group with regard to the visual stimuli (see Fig. 3A; stimulus $\times$ group, $\left.F_{(1,21)}=4.70, P<0.05\right)$, but not the auditory stimuli (stimulus $\times$ group, $F_{(1,21)}=1.05$, with no main effect of overshadowing with regard to the auditory stimuli, $F<$ 1). Further, analyses of simple main effects of data from responding to the visual stimuli demonstrated that overshadowing was only observed with visual stimuli in saline-infused rats $\left(F_{(1,21)}=\right.$ 4.70, $P<0.05)$, but not in muscimol-infused animals $(F<1)$. These data show that functioning in the PL cortex during conditioning is necessary for animals to demonstrate the overshadowing effect through the use of an attentional mechanism as argued by Mackintosh $(1975,1976$; see supporting analyses of attentional nature of basic overshadowing effect below).

In order to ensure that the PL cortex would not be necessary to express fear once it had been acquired when competition from contextual cues is low, in Experiment $2 \mathrm{~b}$ we also investigated the

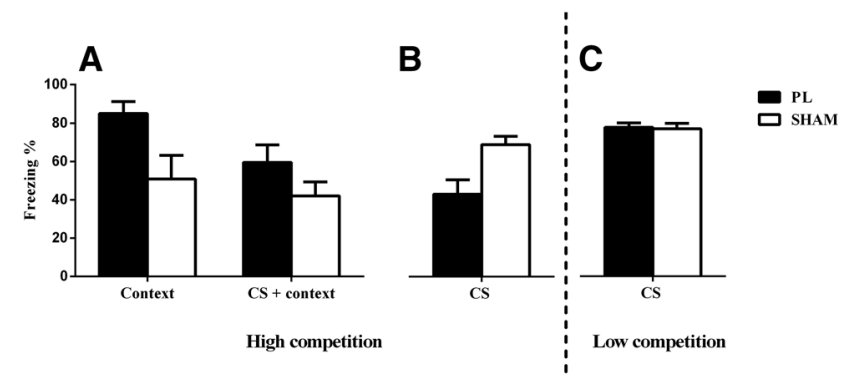

Figure 2. PL lesions only produce a deficit in responding to a CS when there is high competition between contextual and discrete cues. Rates of responding are represented as mean level of observations in which rats spent freezing during CS presentations in the CS test ( \pm SEM). $(A)$ Rats with PL lesions exhibit greater levels of responding to a context when there is high competition between contextual and discrete cues $(B) \mathrm{A}$ deficit in learning about the CS is revealed when rats are tested for levels of responding toward the CS outside of the conditioning context (C) Reducing competition between contextual and discrete cues restores the ability of animals with PL lesions to exhibit fear toward a CS.

impact of muscimol ( $n=11$; Fig. 1B) or saline infusions $(n=11$; Fig. 1B) during the test session in a separate group of rats. Similar to Experiment 2a, we found that rats suppressed leverpress responding during the first presentation of the CSs in the preexposure session (mean suppression ratio $( \pm$ SEM): saline 0.27 (0.05); muscimol 0.20 (0.03), which reduced during the second presentation of the stimuli (mean suppression ratio [ \pm SEM]: saline 0.49 [0.08]; muscimol 0.41 [0.04]). Again, rats gradually reduced lever-press responding during the conditioning session such that they had stopped responding during the second conditioned session during CS presentations (mean suppression ratio [ \pm SEM]: saline 0.16 [0.1]; muscimol 0.00 [0.00]). During extinction test sessions, we found that saline-infused animals exhibited high levels of suppression to both auditory stimuli (mean suppression ratio $[ \pm$ SEM]: compound 0.10 [0.04]; element 0.09 [0.03]) similarly to muscimol-infused animals (mean suppression ratio [ \pm SEM]: compound 0.10 [0.03]; element 0.11 [0.05]). All animals exhibited high levels of suppression toward the visual stimulus trained individually with the outcome relative to the overshadowed visual stimulus conditioned in compound. This was confirmed by statistical analyses, where a mixed-design repeated-measures ANOVA on responding to the visual stimuli revealed a main effect of stimulus (see Fig. 3B; $F_{(1,20)}=8.12, P<0.05$ ), with no between-group interaction (stimulus $\times$ group, $F<1$ ). Further, a mixed-design repeated-measures ANOVA of the data from the auditory stimuli demonstrated there was no main effect of stimulus, demonstrating that animals did not show overshadowing in regards to the auditory stimuli $(F<1)$. A formal analysis of the basic overshadowing effect in animals given saline infusions in both Experiment $2 \mathrm{a}$ and $2 \mathrm{~b}$ demonstrated that this effect was attentional in nature. That is, we conducted a repeated-measures ANOVA on animals' responses to both the visual and auditory stimuli during the test session. This analysis revealed a significant main effect of modality $\left(F_{(1,21)}=24.48, P<0.05\right)$, a significant overshadowing effect (i.e., compound versus element; $F_{(1,21)}=9.40$, $P<0.05)$, and a significant interaction between these factors $\left(F_{(1,21)}=5.24, P<0.05\right)$. Analyses of simple main effects demonstrated that the source of this interaction was due to a significant overshadowing effect in regards to the visual stimuli $\left(F_{(1,21)}=\right.$ 13.01, $P<0.05)$, but not for the auditory stimuli $(F<1)$. A demonstration of overshadowing solely in the visual stimuli is consistent with a Mackintosh (1975) interpretation of the effect, where attention is down-regulated toward the less salient element of the compound, rather than a distribution of learning across 
both stimuli conditioned in compound (i.e., a US-processing mechanism; Rescorla and Wagner 1972). Taken together, these data show that the PL cortex is involved in modulating attention toward cues during learning but not the expression of previously acquired fear when competition from contextual cues is low. These data call for a refinement of the view that the PL cortex promotes the expression of conditioned fear, suggesting that a role for the PL cortex in fear expression is dependent on a role in directing attention toward relevant cues based on how well they predict an outcome, in line with appetitive findings (Sharpe and Killcross 2014).

It is worth noting here that others have found that posttraining inactivation of the PL cortex results in a reduction of fear responding toward the CS (Corcoran and Quirk 2007), in contrast to the present results. We would raise the possibility that this finding occurred as the result of a failure to modulate attention toward predictive cues and overcome competition between contextual and discrete cues during the test session. That is, just as attention can change what is learnt, the amount of attention directed toward a stimulus will also determine the level of conditioned responding it will support (Mackintosh 1975; Le Pelley 2004; Pearce and Mackintosh 2010). For example, in the Corcoran and Quirk (2007) study, animals received conditioning during a single training session, receiving seven CS-US pairings with an ITI varying about a 3 -min mean. This would be likely to have promoted high competition between contextual and discrete cues. With the PL cortex functioning normally during conditioning, animals could still learn that the CS is the better predictor relative to the context. However, this attentional modulation is dependent on the functioning of the PL, so when the PL cortex is then inactivated at test, these animals fail to attend selectively toward the CS (based on a failure of the ability of the CS's predictive history to modulate attention), resulting in a conditioned response that is equally derived from the associative strengths of the CS and context and is therefore reduced overall. More specifically, when the PL cortex is inactivated during the test session, strong, unmodulated competition between contextual and discrete cues will mean that animals will direct a relatively smaller amount of attention toward the CS, compared with the attention it would usually merit based on its training history. As the CS accrued the greatest majority of associative strength during training, this will result in low levels of fear as attention is directed toward contextual cues that do not possess high associative strength.

In support of this view, we found no effect of PL inactivation on the ability of animals to express fear to a CS when animals had received multiple pretraining exposures to the conditioning
A

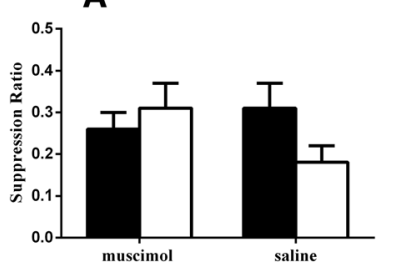

B

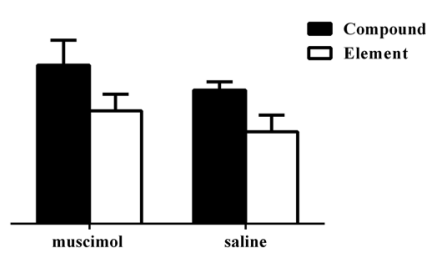

Figure 3. PL inactivation during conditioning disrupted subsequent exhibition of the overshadowing effect, while PL inactivation at test was without effect. Rates of responding are represented as suppression ratios with error bars representing the standard error of the mean $(A)$ Animals receiving saline infusions during conditioning exhibited lower levels of fear to stimuli conditioned in compound relative to stimuli individually paired with the outcome, indicative of an overshadowing effect. Animals receiving infusions of the muscimol into the PL cortex failed to demonstrate this difference. $(B)$ Rats receiving either saline or muscimol infusions during the test session demonstrated the overshadowing effect. context and the test session used lengthy ITIs. Importantly, the processes that result in this decreased competition (such as increased habituation) are not those that depend on the active modulation of attention based on predictive history by the PL cortex (Dias and Honey 2002). That is, the PL cortex is not involved in the bottom-up process which allows a reduction in the salience of a stimulus with repeated exposure in the absence of an outcome. Rather, we suggest the PL cortex is involved in a top-down process which allows an attentional response toward a stimulus to change as a result of the degree to which it predicts an outcome relative to other present stimuli. In the overshadowing procedure used here, critical tests were of a single CS and hence attentional modulation (in the absence of strong competition from contextual cues) was not a factor at test, and PL inactivation was therefore without effect. These data suggest that the PL cortex is only required when there is direct competition between stimuli, supporting an active role for this region in directing attention (and hence responding) toward predictive cues rather than in the production of conditioned responding per se. In line with this interpretation, we would argue that data demonstrating PL neurons are active during CS presentation (Burgos-Robles et al. 2009) is indicative of the maintenance of an attentional response toward relevant stimuli in order to generate appropriate responding.

Formally, a deficit in overshadowing may not only result from an impairment in modulating attention toward cues. Models of associative learning which assume that a set amount of learning supported by the US is distributed across all present cues on the basis of their salience, need not appeal to attentional processes to explain overshadowing (Rescorla and Wagner 1972). However, we have conducted a number of experiments in our laboratory that have confirmed the attentional nature of the PL deficit in an appetitive setting (Sharpe and Killcross 2014). More specifically, we have demonstrated that animals with PL lesions were capable of blocking learning about a stimulus when it was presented simultaneously with another stimulus that already predicted the outcome. However, in a subsequent test, PL-lesioned animals learnt about this blocked cue more rapidly than shamlesioned animals when the stimulus alone was paired with reinforcement, suggesting these animals did not change the degree of attention directed toward the blocked cue despite the fact that it was a redundant predictor of the outcome. This indicated that these animals are capable of distributing learning across stimuli paired with an outcome in compound, but have a specific deficit in changing the degree of attention toward these cues. This demonstrates that the PL cortex produces an attentional deficit, which disrupts exhibition of the overshadowing effect, rather than a more general deficit in distributing a finite amount of learning across multiple cues.

A role for attentional processes in the demonstration of the overshadowing effect is also supported in the data in the present experiments. Consistent with Mackintosh's (1975) model of attention, we found that all animals exhibited high levels of learning about auditory stimuli whether they were conditioned in compound or as an element during training, whereas animals exhibiting the overshadowing effect only did so in relation to the less salient visual cues (i.e., exhibited less learning about the visual stimulus conditioned in compound). This observation is only predicted by an attentional account of overshadowing, where attention is down-regulated toward the less salient element of the compound, in this case the visual stimulus (Mackintosh $1975,1976)$. This is not predicted by a model which used an errorcorrection mechanism to account for the overshadowing effect (such as Rescorla and Wagner 1972). As these models predict overshadowing via the presence of the summed-error term, where the finite amount of learning that can be supported by a US must be distributed across all present cues, use of a US-processing 
mechanism should result in the exhibition of mutual overshadowing where learning about both stimuli (auditory or visual) conditioned in compound is reduced relative to those which are conditioned alone. Thus, the nature of the effect observed suggests that the procedure used in the present experiments elicited the use of an attentional mechanism and, therefore, a deficit in overshadowing was the result of a disruption of this process.

As a final point, it is worth pointing out that an involvement of the PL cortex in fear learning but not expression in the present experiments is at odds with our recent findings assessing the impact of PL inactivation in a contextual bi-conditional discrimination (Sharpe and Killcross 2015). Here, we gave animals two CSs in two different contexts. In context A, CSA predicted shock and CSB was presented without shock. In context $B$, these contingencies were reversed so that CSB was now paired with shock and CSA was presented without shock. Animals given saline infusions during either conditioning or test demonstrated high levels of fear to CSA in context A, and CSB in context B. However, these animals did not show fear toward these CSs when they were presented in the context where they were not paired with shock. In contrast, we found that animals given muscimol infusions into the PL cortex during either conditioning or test failed to demonstrate context-specific responding. We believe the involvement of the PL cortex in responding in this paradigm (and not the present experiments) owes to the need for response modulation during both conditioning and test in the contextual bi-conditional discrimination. That is, as the CSs are ambiguous during both phases of the experiment, the PL cortex is necessary to allow animals to use the contextual cues to disambiguate the significance of the CS at both time points. We believe this reflects a general role for the PL cortex in the use of higher-order cues (e.g., the associative history of a stimulus or contextual cues) to modulate responding. In the present experiments, this role was only necessary during conditioning when animals need to use the predictive history of the stimuli conditioned in compound to modulate attentional responding (and hence learning) to demonstrate the overshadowing effect. This was not the case in the test phase of the overshadowing procedure where all stimuli were presented individually and competition from contextual cues remained low. Thus, we would argue that the PL cortex is not necessarily involved in fear learning or expression per se but, rather, using higher-order cues to modulate responding toward cues. The involvement of the PL cortex in fear learning or expression will, therefore, depend on the task at hand.

A role for the PL cortex in restricting learning to predictive cues is consistent with a role for this region in promoting behavioral flexibility. In addition to modulating attention, the PL cortex has also been implicated in the resolution of response conflict, the promotion of goal-directed behaviours, strategy switching, and response monitoring (Balleine and Dickinson 1998; Gisquet-Verrier and Delatour 2006; Floresco et al. 2008 Marquis et al. 2007). All of these tasks require use of higher-order information such as goal value, task demands, and contextual stimuli to influence the way an animal responds to ambiguity. In the context of the present experiments, the PL cortex uses the associative history of a stimulus to change the attentional response elicited by a stimulus. Such research suggests the PL cortex utilizes information in the environment to endow an animal with a level of behavioral complexity that affords it flexibility in the face of uncertain situations.

\section{Acknowledgments}

These data were funded by a project grant from the National Health and Medical Research Council awarded to S.K.

\section{References}

Balleine BW, Dickinson A. 1998. Goal-directed instrumental action: contingency and incentive learning and their cortical substrates. Neuropharmacology 37: 407-419.

Birrell JM, Brown VJ. 2000. Medial frontal cortex mediates perceptual attentional set shifting in the rat. J Neurosci 20: 4320-4324.

Burgos-Robles A, Vidal-Gonzalez I, Quirk GJ. 2009. Sustained conditioned responses in prelimbic prefrontal neurons are correlated with fear expression and extinction failure. J Neurosci 29: 8474-8482.

Corcoran KA, Quirk GJ. 2007. Activity in prelimbic cortex is necessary for the expression of learned, but not innate, fears. J Neurosci 27: 840-844.

Dias R, Honey RC. 2002. Involvement of the rat medial prefrontal cortex in novelty detection. Behav Neurosci 116: 498-503.

Floresco SB, Block AE, Tse MTL. 2008. Inactivation of the medial prefrontal cortex of the rat impairs strategy set-shifting, but not reversal learning, using a novel, automated procedure. Behav Brain Res 190: 85-96.

Gisquet-Verrier P, Delatour B. 2006. The role of the rat prelimbic/infralimbic cortex in working memory: not involved in the short-term maintenance but in monitoring and processing functions. Neuroscience 141: 585-596.

Holland PC. 2000. Trial and intertrial durations in appetitive conditioning in rats. Anim Learn Behav 28: 121-135.

Holson RR. 1986. Mesial prefrontal cortical lesions and timidity in rats. I. Reactivity to aversive stimuli. Physiol Behav 37: 221-230.

Lacroix L, Spinelli S, Heidbreder CA, Feldon J. 2000. Differential role of the medial and lateral prefrontal cortices in fear and anxiety. Behav Neurosci 114: $1119-1130$.

Le Pelley ME. 2004. The role of associative history in models of associative learning: a selective review and a hybrid model. QJ Exp Psychol B 57: $193-243$.

Mackintosh NJ. 1975. A theory of attention: variations in the associability of stimuli with reinforcement. Psychol Rev 82: 276-298.

Mackintosh NJ. 1976. Overshadowing and stimulus intensity. Anim Learn Behav 4: $186-192$.

Mais JHR, Vossen JMH. 1993. Competition for associative strength between a punctate signal and contextual stimuli: effect of signal preexposure versus context preexposure. Behav Processes 30: 29-46.

Marquis JP, Killcross S, Haddon JE. 2007. Inactivation of the prelimbic, but not infralimbic, prefrontal cortex impairs the contextual control of response conflict in rats. Eur J Neurosci 25: 559-566.

McAllister WR, McAllistair DE, Weldin GH. 1974. Intertrial interval effects in classically conditioning fear to a discrete conditioned stimulus and to situational cues. J Comp Physiol Psychol 87: 582-590.

Morgan MA, LeDoux JE. 1995. Differential contribution of dorsal and ventral medial prefrontal cortex to the acquisition and extinction of conditioned fear. Behav Neurosci 109: 681-688.

Paxinos G, Watson C. 1998. The rat brain in stereotaxic coordinates. San Diego, CA: Academic press.

Pearce JM, Mackintosh NJ. 2010. Two theories of attention: a review and possible integration. In Attention and associative learning (ed. Mitchell CJ, Le Pelley ME), pp. 351-385. Oxford University Press, New York.

Rescorla RA. 1984. Signaling inter-trial shocks attenuates their negative effect on conditioned suppression. Bull Psychon Soc 22: 225-228.

Rescorla RA, Wagner AR. 1972. A theory of Pavlovian conditioning: variations in the effectiveness of reinforcement and non-reinforcement. In Classical conditioning II: current research and theory (ed. Black AH, Prokasy EF), pp. 64-99. Appleton-Century-Crofts, New York.

Rhodes SE, Killcross S. 2007. Lesions of rat infralimbic cortex enhance recovery and reinstatement of an appetitive Pavlovian response. Learn Mem 11: 611-616.

Sharpe MJ, Killcross S. 2014. The prelimbic cortex contributes to the down-regulation of attention toward redundant cues. Cereb Cortex 24: 1066-1074.

Sharpe MJ, Killcross S. 2015. The prelimbic cortex uses higher-order cues to modulate both the acquisition and expression of conditioned fear. Front Syst Neurosci 8: 235.

Sierra-Mercado D, Padilla-Coreano N, Quirk GJ. 2011. Dissociable roles of prelimbic and infralimbic cortices, ventral hippocampus, and basolateral amygdala in the expression and extinction of conditioned fear. Neuropsychopharmacology 36: 529-538.

Received January 22, 2015; accepted in revised form March 25, 2015. 


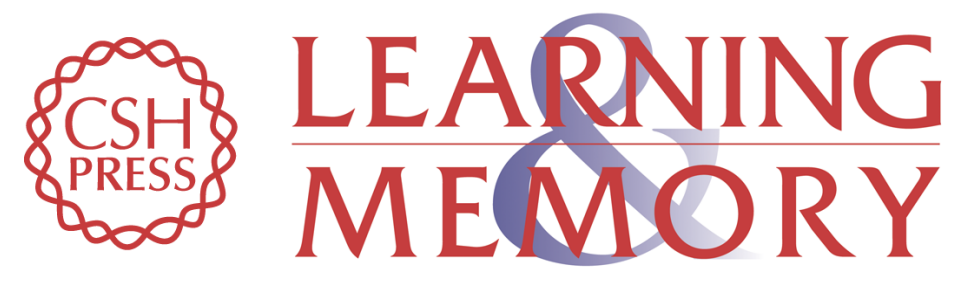

\section{The prelimbic cortex directs attention toward predictive cues during fear learning}

Melissa J. Sharpe and Simon Killcross

Learn. Mem. 2015, 22:

Access the most recent version at doi:10.1101/lm.038273.115

References This article cites 22 articles, 3 of which can be accessed free at:

http://learnmem.cshlp.org/content/22/6/289.full.html\#ref-list-1

Creative This article is distributed exclusively by Cold Spring Harbor Laboratory Press for the

Commons first 12 months after the full-issue publication date (see

License http://learnmem.cshlp.org/site/misc/terms.xhtml). After 12 months, it is available under a Creative Commons License (Attribution-NonCommercial 4.0 International), as described at http://creativecommons.org/licenses/by-nc/4.0/.

Email Alerting Receive free email alerts when new articles cite this article - sign up in the box at the Service top right corner of the article or click here. 